\section{Top universal composite 12 years running}

Estelite Sigma Quick has been awarded the Dental Advisor Top Product Universal Composite for the $12^{\text {th }}$ year running.

Featuring Tokuyama's patented RAP monomer and Aesthetic 'Pearl' technology, Estelite Sigma Quick delivers an extended working time in ambient light yet cures in only ten seconds. There is also less residual monomer and minimal after-cure colour change for long-term aesthetic satisfaction.

In addition, Estelite Sigma Quick offers miracle shade matching. Because of its unique Aesthetic 'Pearls' it offers inherent shade mimicking so that, in most cases, just one shade will blend perfectly with the natural teeth leaving invisible margins and undetectable restorations. These 'Pearls' also facilitate outstanding polishability and a lustre that lasts and lasts, allied to high wear resistance and very low abrasion of the opposing teeth.

Estelite Sigma Quick is ideally used in combination with Tokuyama's award-winning Universal Bond which can be used to bond all direct and indirect restorative materials, Garrison Sectional Matrix Systems and for the ultimate aesthetic finish Eve polishers.

If you prefer layering techniques Tokuyama's Asteria delivers outstanding results which can be characterised using Estelite Color.

For more information, call Trycare Ltd on 01274885544 or visit www.trycare.co.uk/estelite.

\section{Start implementing simple implant treatment plans}

The Quest Implantology Course 2022 teaches delegates everything they need to start implementing simple implant treatment plans plus 36 hours of CPD.

Royal College of Surgeons of Edinburgh accredited and presented by Girish Bharadwaj, the course gives delegates a thorough basic knowledge of implantology including an overall perspective with an evidence-based approach; necessary surgical and restorative skills to implement simple treatment plans; an understanding of the challenges involved in the surgical and prosthetic phases of treatment; and an understanding of when to refer based upon their skill levels.

Completion of the course will enable participants to diagnose and carry out implant treatment planning; carry out initial risk assessment and consent the patient appropriately; insert and restore implants under supervision in a simulated environment; identify the complexity of individual cases and place implants in simple cases under supervision; and know when to refer for additional support and guidance.

Held in Edinburgh, course fees are $£ 1,250.00$ (including VAT) per module and $£ 3,500.00$ (including VAT) for the full course.

Delegates attending all three modules will receive a Touareg $\mathrm{S}$ Advanced Surgical Kit, an RS8032 Tip Drill and FIVE Touareg S Implants total value $£ 1,339.00$ plus VAT FREE.

For further information or to book your place contact info@ ochilviewdental.co.uk.

\section{Are you ready to go?}

Align Technology, Inc. has announced the launch of Go Day to enable general dentists to discover more about its Invisalign Go portfolio. The online event runs from $7.00 \mathrm{pm}$ on 24 February to give general dentists the opportunity to explore how Invisalign clear aligners and a digital workflow can help them provide comprehensive dental care with improved aesthetic and oral health outcomes, as well as a better patient experience.

This free, online event covers these topics with the help of two experts who will explore how the integration of the Invisalign Go system with additive prosthetics provides a minimally invasive treatment approach, and how the minimally invasive approach of the Invisalign Go Plus system helps GDPs achieve long-lasting treatment in a short timeframe.

Professor Camillo D’Arcangelo will present 'Non-invasive, simple and predictable dentistry for aesthetic and functional rehabilitation', and Dr Camilla Morrison will speak on 'Minimally invasive dentistry and the integration with Invisalign Go Plus'.

Align Technology's Jody Carter, Senior Director, Marketing GP Channel EMEA, Abhishek Ganguly VP, Sales GP Channel EMEA, and Lee Taylor, Vice President of Marketing EMEA will introduce the Invisalign Go system and provide information on the Invisalign Go portfolio, including Invisalign Go Plus. After a speaker discussion, the event will be concluded by Align's Senior VP and Managing Director, EMEA Markus Sebastian.

The event is open to Invisalign providers and non-providers and will be presented in English, with online translations available in Italian, German, French, Polish and Spanish.

To register visit: http://www.invisalign.eu/provider/gp/ professional-development/educational-events/ 3GUORFgQBx0byKIqhXD8FG.

\section{Could your patients benefit?}

The Biosmalto Impact Action Mousse utilises an innovative formulation to deliver rapid remineralisation benefits, while protecting the teeth from further damage through acid erosion, acid attacks, mechanical abrasion and sensitivity.

It can be used as part of therapy to treat carious lesions, tooth wear, dry mouth, white spots, molar or incisor demineralisation, fluorosis or sensitivity (especially pre- and post-air polishing).

The chairside application of Biosmalto provides an additional service for patients, with a $50 \mathrm{ml}$ tube available for patients to take home. SLSfree, easy to apply, vegan and translucent, it is also suitable for patients with lactose intolerance and can be used with or without trays.

Want to know more? Contact the expert team at J\&S Davis to support your patients with Biosmalto today.

For more information on the industry-leading products available from J\&S Davis, visit www.js-davis.co.uk, call 01438747344 or email jsdsales@js-davis.co.uk. 